\title{
Nuclear Genome Size Determination Of Christia Vespertilionis Via Flow Cytometry
}

\section{Mohd Razik Midin*, Muhammad Irfan Fikri \& Siti Sarah Zailani}

Department of Plant Science, Kulliyyah of Science, International Islamic University Malaysia, Jalan Sultan Ahmad Shah, Bandar Indera Mahkota, 25200 Kuantan, Pahang, Malaysia

*Corresponding author e-mail: mohdrazik@iium.edu.my

\section{DOI: 10.2478/acmy-2020-0012}

\begin{abstract}
:
Christia vespertilionis (butterfly wing plant) is an ornamental plant originated from South East Asia with reported usage in traditional medicine practice and potential as an anticancer and antitumor. This research aims to estimate the genome size of C. vespertilionis via flow cytometry (FCM) method. The research was conducted with the optimisation of nuclear suspension preparation followed by the genome size estimation. Two chopping techniques [manual chopping (MC) and BDTM Medimachine (MM)] and two lysis buffers (Otto and LBO1) were tested. Otto buffer with manual chopping was found to be the most suitable method, generated fine DNA peak with minimum debris background, and coefficient of variation (CV) value less than $3 \%$. Five replicates of the FCM analysis were made for the genome size determination. The estimated genome size of C. vespertilionis was found to be 3.22 pg by using Glycine max cv. Polanka ( $2 \mathrm{C}=2.5 \mathrm{pg}$ ) as an external reference standard. Further comparison with other Christia species was not possible due to the lack of data on genome size. The genome size data of $\mathrm{C}$. vespertilionis can be useful for future morphology and genetics studies of Christia species.
\end{abstract}

Keywords: Christia vespertilionis, pokok rerama, flow cytometry, genome size

\subsection{Introduction}

Christia vespertilionis (L. f.) Bakh. f., is commonly known as 'mariposa,' 'butterfly wing plant,' and 'Rerama' in Malaysia. It is a non-climbing perennial herb found in South East Asia and known for its uniquely shaped leaves resembling a pair of butterfly wings, hence its nickname. $C$. vespertilionis is being cultivated widely as a garden and ornamental plant. The leaves of $C$. vespertilionis can be used for medicinal purposes such as treating snake bites, tuberculosis, heal bone fractures, increase blood circulation, bronchitis, and cold [1,2]. Many studies focused on the chemical properties and medicinal effects of the plant, increasing its potential to be incorporated with modern medicines such as antiplasmodial agents against Plasmodium falciparum, a major cause of malaria [3]. Previous studies discussed the potential of the plant as an anticancer based on its antiproliferative activity against human neuroendocrine tumor cells [4,5]. Recent studies reported on the total phenolic compound and antioxidant scavenging capacity of the plant's extract [6].

The genome size of individual plant species is important in many research fields, including taxonomy, phylogeny, and evolutionary changes, as well as in biotechnology, especially for gene cloning planning and genome sequencing projects [7]. Genome size is the total amount of DNA in the nucleus of an organism that is measured either in picograms $\left(1 \mathrm{pg}=10^{-12} \mathrm{~g}\right.$ ) or megabase pairs (Mbp, with $1 \mathrm{pg}=978 \mathrm{Mbp}$ ) [8,9]. Flow cytometry has become the method of choice for genome size determination as it can analyse thousands of particles per second, requires small tissue samples, and finally produced high accuracy and resolution data [10-13].

Previous studies documented the potential of $C$. vespertilionis for both modern and traditional medicine. However, its genome size information is still lacking. The data of genome size is important for other research areas, such as taxonomy, evolutionary studies, and genome sequencing. Up to now, there is only genome size data of one Christia species, C. obcordata has been reported [14]. Due to this, this study aims to optimize the nuclear suspension method prior to FCM analysis as well as determine the genome size of $C$. vespertilionis.

\subsection{Material and Methods}

\subsection{Plant Material}

C. vespertilionis seedlings were obtained from the nursery of International Islamic University Malaysia (IIUM) Kuantan, Pahang, and utilized for analysis. For nuclei suspension preparation, young leaves were selected as they were softer and more comfortable to process and also contained less secondary metabolites [15].

\subsection{Nuclei suspension preparation}

Fresh leaf samples of $C$. vespertilionis were cut into three small pieces and placed in a petri dish with nuclear lysis buffer. Nuclei were extracted from young leaves using two methods, which are manual chopping (MC) and BD ${ }^{\mathrm{TM}}$ Medimachine blender (MM) [16,17]. Two nuclear lysis buffers, namely LBO1 and Otto buffers supplemented with RNaseA, propidium iodide (PI), and reducing agents mercaptoethanol were used [18-20]. The suspension of released nuclei was filtered into a $10 \mathrm{~mL}$ falcon tube through $0.22 \mu \mathrm{m}$ filter and incubated for 10 minutes at room temperature to allow optimum PI intercalation with the DNA strands. PI fluorochrome was selected for this analysis because it has been proven to produce a more precise fluorescent intensity histogram peak with lower CV and able to intercalate the entire DNA region [21].

\subsection{FCM analysis}

Stained nuclei suspension samples were analysed by using FACSCalibur FCM (BD Biosciences, San Jose, CA) equipped with $15 \mathrm{~mW}$ argon ion laser at 488 $\mathrm{nm}$. CellQuest software (Beckton Dickinson) incorporated into the FCM machine was used to measure the relative fluorescence DNA of histogram peak and CV value. Histograms were collected over 1024 channels, and 10,000 events were captured for each sample.

\subsection{Genome size measurement}

For genome size determination, Glycine max cv. Polanka (Soybean, 2C=2.5 pg; seeds were provided by J Doležel, Olomouc University) was used as an external reference standard $(17,22)$. Fluorescence intensity of all samples was compared with fluorescence intensity of soybean variety Polanka to obtain the ratio, then multiplied by the genome size of soybean, $2 \mathrm{C}=2.5 \mathrm{pg}$. The values of fluorescent intensity peaks and genome size of samples were analysed using CellQuest3R.

\subsection{Results and Discussion}

\subsection{Optimization of nuclei preparation}

The quality of nuclei suspension is determined by analyzing the histogram DNA peak prior to FCM measurement. Good histogram DNA peak must have (1) minimal debris background, (2) symmetrical DNA peak, and (3) low CV value. In this study, two lysis buffers, namely Otto and LBO1 with two chopping methods, manual chopping (MC), and Medimachine (MM), were used for FCM analysis sample preparation. Figure 1 and Table 1 shows the histogram of the nuclei fluorescence intensities obtained as well as CV value from all four preparation methods used 

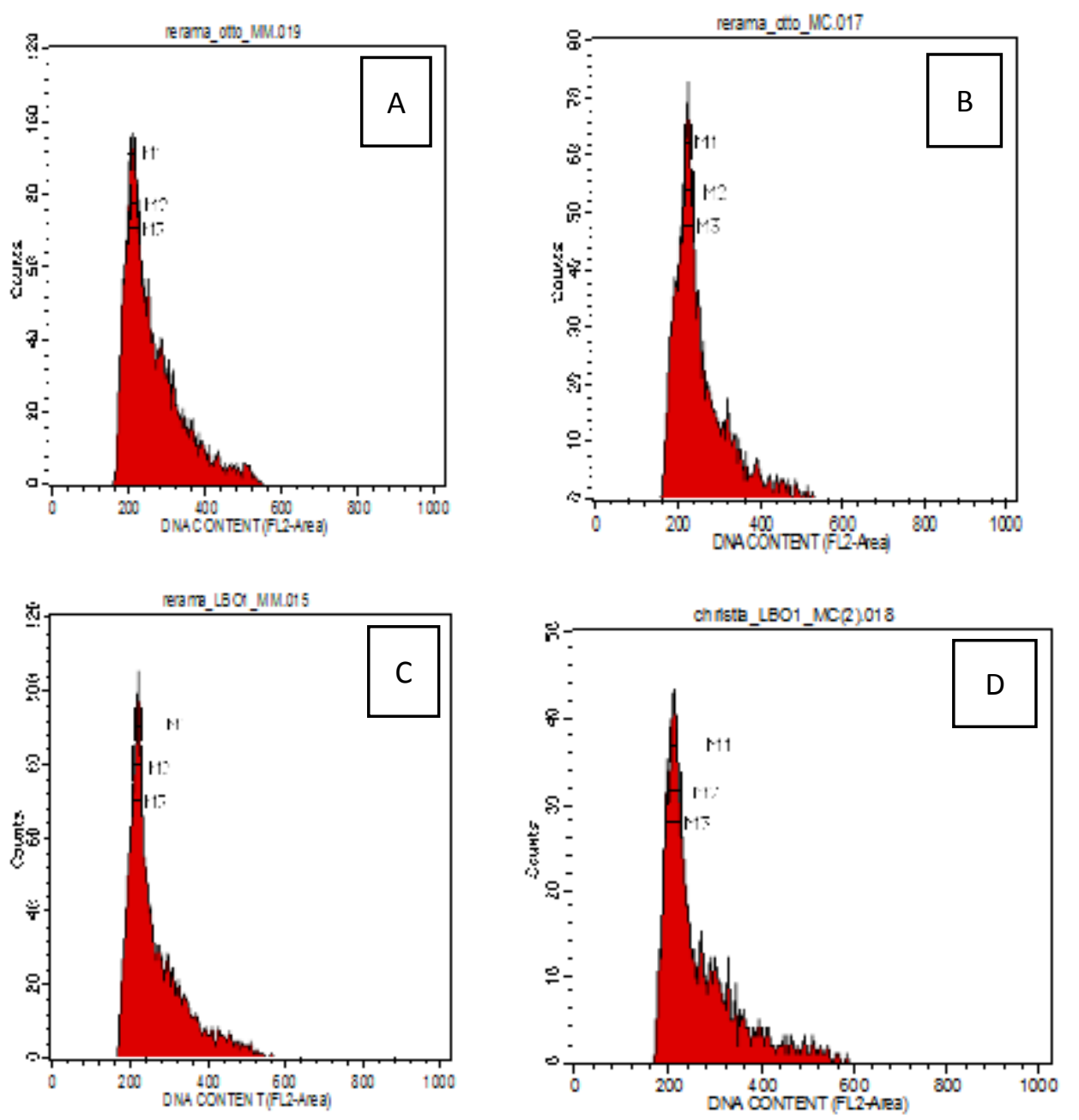

Figure 1: Histograms of nuclei fluorescence intensity:(A) Otto-MM, (B) Otto-MC, (C) LBO1-MM and (D) LBO1-MC

Table 1: CV value obtained by all four nuclei preparation methods used

\begin{tabular}{ccc}
\hline Histogram & Lysis buffer and chopping method & Average CV \\
\hline A & Otto-MM & 3.053 \\
B & Otto-MC & 2.667 \\
C & LBO1-MM & 2.703 \\
D & LBO1-MC & 3.450 \\
\hline
\end{tabular}

By comparing the histogram peaks of all methods used in nuclei preparations, both Otto and LBO1 lysis buffers are found suitable for FCM analysis as they generated fine histogram DNA peaks. Histogram DNA peak of the MM method produced slightly more debris background as compared to the MC method, which can be caused by uncontrolled chopping by the machine. The otto-MC method generated histogram B with less debris background, and the lowest $\mathrm{CV}$ value was chosen for the genome size determination of $C$. vespertilionis.

Too much chopping can break the nuclei and produce debris background on DNA histogram. FCM analysis demonstrates higher debris background obtained from the MM method because of uncontrolled chopping, which can cause more nuclei breakage. In this study, the MC method shows a considerable amount of debris, despite showing lower debris background as compared to MM. This problem can be minimized by reducing the vigour of chopping and using a sharp scalpel. Besides, the staining incubation period can be optimized to minimize the possibility of debris peak to occur. Debris can also be removed from histograms by a combination of electronic thresholding and right gating strategies.

Both Otto and LBO1 lysis buffers generated fine DNA histogram peaks and CV values less than 5\%. Different nuclear isolation buffer can cause different effects on numbers of nuclei in the sample, the amount of debris, and peak broadness. The result shows little difference in peak sharpness and debris amount for both buffers, indicating both buffers are suitable for FCM analysis on $C$. vespertilionis. However, all DNA histogram peaks have a broad base, which might be because of the negative effect of cytosol. Several steps can be applied in reducing the cytosolic effect: (1) Add reducing agents such as mercaptoethanol and the tannin-binder PVP-10/PVP-40, (2) reduce vigor of chopping, (3) test a different tissue, (4) use a different nuclear isolation buffer, and (5) prepare the samples in a walk-in cold room.

The selection of DNA fluorochrome for DNA staining is another crucial step in sample preparation for FCM analysis. DAPI and PI are the most popular DNA fluorochromes. However, DAPI is base selective, hence may cause errors in genome size estimation [22]. Thus, PI was used in this study as it binds to the entire DNA region as well as produce a DNA peak with a lower CV.

\subsection{Genome size determination}

Glycine max cv. Polanka was used as an external reference standard due to having established genome size, $2.5 \mathrm{pg}$, and soft leaves structure, which helps to ease the sample preparation [23]. An ideal reference standard for genome size estimation should be known and neither too close nor too distant to the target species to avoid the risk of non-linearity and offset errors, which can cause overlapping DNA peak of the standard and the sample. Due to this, the external reference standard method was chosen in this study to avoid DNA peak overlap [24]. 
Several requirements are needed to be fulfilled for reliable genome size determination such as (1) extraction of sufficient quantity of nuclei with undegraded DNA using suitable lysis buffer, (2) stoichiometric DNA staining, (3) awareness to secondary metabolites interferences during DNA staining, (4) ribonuclease treatment prior sample analysis and (5) selection of ideal reference standard with established genome size. All these requirements have been fulfilled in this study; thus, the genome size of $C$. vespertilionis was successfully estimated.

This report is the first on the genome size of $C$. vespertilionis, which was found to be $3.22 \mathrm{pg}$. The first reported genome size of a Christia species was $C$. obcordata, with the genome size of $0.65 \mathrm{pg}$. The estimated genome size of $C$. vespertilionis was found to be about five times higher than that of $C$. obcordata. As C. obcordata was the only Christia species reported with estimated genome size, comparison with other Christia species was not possible. Knowledge of the genome size of Christia species is vital in order to determine interspecific and intraspecific genome size variation. Intraspecific and interspecific genome size variation provides essential information on the evolution of the genome and taxonomy as an indicator of taxonomic heterogeneity [25]. The understanding of genome size variation may contribute to evolutionary theory; for example, it has been suggested that younger taxa have relatively more considerable intraspecific genome size variation than older ones [26]. In taxonomy, intraspecific genome size variation provides evidence for the presence of more than one taxonomic entity within a species [27].

\subsection{Conclusion}

In summary, the genome size of $C$. vespertilionis was successfully measured by the FCM method. The data obtained can be used as a reference for future studies. C. vespertilionis is a promising plant with many reports on its potential as a medicine for critical diseases. As a well-known ornamental plant and medicinal plant with high potential value, the cytogenetics study of $C$. vespertilionis can be beneficial and useful for future studies. It is recommended to conduct further cytogenetics studies such as chromosome number determination and physical mapping on this species. The genome size and chromosome number data of this species will contribute to the species classification as well as future genome sequencing projects.

\subsection{References}

[1] J. Nguyen-Pouplin, H. Tran, H. Tran, T.A. Phan, C. Dolecek, J. Farrar, T.H. Tran, P. Caron, B. Bodo, P. Grellier, "Antimalarial and cytotoxic activities of ethnopharmacologically selected medicinal plants from South Vietnam", Journal of Ethnopharmacology, Vol. 109, No. 3, Pp. 417-427, 2007.

[2] S. Ganesan, S.F. Sabran, M.H. Mazlun, "Plant Diversity Assessment and Traditional Knowledge Documentation of Home Gardens in Parit Raja, Batu Pahat, Johor", In IOP Conference Series: Earth and Environmental Science, Vol. 269, No. 1, 012018, 2019. IOP Publishing.

[3] H.C. Upadhyay, B.S. Sisodia, H.S. Cheema, J. Agrawal, A. Pal, M.P. Darokar, S.K. Srivastava, "Novel Antiplasmodial Agents from Christia vespertilionis", Natural Product Communications, 2013. https://doi.org/10.1177/ 1934578X1300801123

[4] S.N. Bunawan, B. Hamidun, S.N. Baharum, "The red butterfly wing (Christia vespertilionis): A promising cancer cure in Malaysia", International Journal of Pharmacy and Pharmaceutical Sciences, Vol. 7, No. 8, Pp. 5, 2015.

[5] D. Hofer, G. Schwach, N. Ghaffari Tabrizi-Wizsy, A. Sadjak, S. Sturm, H. Stuppner, R. Pfragner, "Christia vespertilionis plant extracts as novel antiproliferative agent against human neuroendocrine tumor cells", Oncology reports, Vol. 29, No. 6, Pp. 2219-2226, 2013.

[6] H. Mat Jusoh, N. Haron, "The total phenolic contents and antioxidant scavenging capacity in free and bound extracts of Christia Vespertilionisis (Daun Rerama)", International Journal of Allied Health Sciences, Vol. 3, No. 2, Pp. 649-657, 2019.

[7] J. Doležel, J.A.N. Bartoš, “Plant DNA flow cytometry and estimation of nuclear genome size”, Annals of botany, Vol. 95, No. 1, Pp. 99-110, 2005.

[8] J. Doležel, J. Bartos, H. Voglmayr, J. Greilhuber, "Nuclear DNA content and genome size of trout and human. Cytometry", Part A: the journal of the International Society for Analytical Cytology, Vol. 51, No. 2, Pp. 127-8, 2003.

[9] R. DeSalle, T.R. Gregory, J.S. Johnston, "Preparation of Samples for Comparative Studies of Arthropod Chromosomes: Visualization, In Situ Hybridization, and Genome Size Estimation", Methods in Enzymology, Vol. 395, Pp. 460-488, 2005.

[10] M.D. Bennett, I.J. Leitch. “Nuclear DNA amounts in angiosperms: Targets, trends and tomorrow”, Annals of Botany, Vol. 107, No. 3, Pp. 467-590, 2011.

[11] D.W. Galbraith, G.M. Lambert, "High throughput monitoring of plant nuclear DNA contents via flow cytometry”, Methods in Molecular Biology, Vol. 918, Pp. 311-325, 2012.

[12] J. Vrána, P. Cápal, M. Bednářová, J. Doležel, "Flow cytometry in plant research: A success story”, In: Nick P, Opatrny Z (eds) Applied Plant Cell Biology, Plant Cell Monograph 22. Springer, Berlin Heidelberg New York, Pp. 395-429, 2014.

[13] G.M.A. Carvalho, C.R. Carvalho, F.A.F. Soares, "Flow cytometry and cytogenetic tools in eucalypts: genome size variation $\times$ karyotype stability", Tree Genetics and Genomes, Vol. 13, Pp. 1-11, 2017.

[14] H.F. Sakhanokho, N. Islam-Faridi, "Nuclear DNA content, base composition and cytogenetic characterization of Christia obcordate", Journal of the American Society for Horticultural Science, Vol. 138, No. 3, Pp. 205-209, 2013.

[15] I. Jedrzejczyk, E. Sliwinska, "Leaves and seeds as materials for flow cytometric estimation of the genome size of 11 rosaceae woody species containing DNA-Staining inhibitors", Journal of Botany, Pp. 1-9, 2010.

[16] M.R. Midin, R. Samsul Kamal, A.H. Tarmizi, R. Nulit, M. Madon, "Analysis of oil palm clones, their suspension calli and regenerants via flow cytometry (FCM) and rDNA-fluorescence in situ hybridisation (rDNA-FISH)”, Journal of Oil Palm Research, Vol. 25, No. 3, Pp. 357-367, 2013.

[17] M.R. Midin, M.S. Nordin, M. Madon, M.N. Saleh, H.H. Goh, M.N. Normah, "Determination of the chromosome number and genome size of Garcinia mangostana L. via cytogenetics, flow cytometry and k-mer analyses", Caryologia, Vol. 71, Pp. 35-44, 2018.

[18] J. Doležel, P. Binarova, S. Lucretti, "Analysis of nuclear DNA content in plant cells by flow cytometry", Biologia Plantarum, Vol. 31, Pp. 113-120, 1989.

[19] F. Otto, "Preparation and staining cells for high resolution DNA analysis", In: Radbruch A ed. Flow cytometry and cell sorting. Springer, Berlin Verlag, 101104, 1992.

[20] J. Doležel, W. Göhde, "Sex determination in dioecious plants Melandrium album and M. rubrum using high-resolution flow cytometry", Cytometry, Vol. 19, No. 2, Pp. 103-106, 1995. 
[21] J. Doležel, J. Greilhuber, J. Suda, “Estimation of nuclear DNA content in plants using flow cytometry”, Nature Protocols, Vol. 2, No. 9, Pp. 2233-2244, 2007.

[22] J. Doležel, S. Sgorbati, S. Lucretti, “Comparison of three DNA fluorochromes for flow cytometric estimation of nuclear DNA content in plant”, Physiologia Plantarum, Vol. 85, Pp. 625-631, 1992.

[23] M. Madon, L.Q. Phoon, M.M. Clyde, D.A. Mohd, "Application of flow cytometry for estimation of nuclear DNA content in Elaies", Journal of Oil Palm Research, Vol. 20, Pp. 447-452, 2008.

[24] B. Hendrix, J.M. Stewart, “Estimation of the nuclear DNA content of Gossypium species”, Annals of Botany, Vol. 95, Pp. 789-797, 2005.

[25] M.D. Bennett, I.J. Leitch, “Plant Genome Size Research: A Field In Focus”, Annals of Botany, Vol. 95, Pp. 1-6, 2005.

[26] P. Šmarda, P. Bureš, “Understanding intraspecific variation in genome size in plants”, Preslia, Vol. 82, No. 1, Pp. 41-61, 2010.

[27] B.G. Murray, "When does Intraspecific C-value Variation become Taxonomically Significant”, Annals of Botany, Vol. 95, No. 1, Pp. 119-125, 2005. 